\title{
The Effect of Exposure to Disaster on Cancer Survival
}

\author{
Sue Anne Bell, PhD, FNP-BC ${ }^{1,2}$, Mousumi Banerjee, $P h D^{2,3}$, Jennifer J. Griggs, MD, MPH 2,4, \\ Theodore J. Iwashyna, MD, $P h D^{2,4,5}$, and Matthew A. Davis, MPH, $P h D^{1,2,5}$
}

'University of Michigan School of Nursing , Ann Arbor , MI , USA; ${ }^{2}$ Institute for Healthcare Policy and Innovation, University of Michigan , Ann Arbor , MI , USA; ${ }^{3}$ Department of Biostatistics, University of Michigan School of Public Health , Ann Arbor , MI , USA; ${ }^{4}$ Department of Internal Medicine, University of Michigan Medical School, Ann Arbor , MI , USA; ${ }^{5}$ University of Michigan Institute for Social Research , Ann Arbor , MI , USA.

J Gen Intern Med 35(1):380-2

DOI: $10.1007 / \mathrm{s} 11606-019-05465-\mathrm{x}$

(c) Society of General Internal Medicine 2019

\section{INTRODUCTION}

The frequency and intensity of weather and climate-related disasters that have devastating effects on millions of Americans is expected to increase due to climate change. ${ }^{1,2}$ While the direct death toll of disasters is well-recognized, ${ }^{3}$ little is known regarding how the impact of disasters and their resultant disruption on healthcare affect health outcomes among individuals with serious health conditions and complex healthcare needs. We examined 10-year survival among a large sample of cancer patients diagnosed in areas affected by Hurricane Katrina in 2005.

\section{METHODS}

We used the National Cancer Institute's Surveillance, Epidemiology, and End Results (SEER) registry to examine the relationship between exposure to Hurricane Katrina and 10-year survival. We identified adults diagnosed with invasive breast, colorectal, and lung cancer who received a diagnosis in the six months before Hurricane Katrina's landfall in the fifteen most affected Louisiana parishes. These common cancers were chosen because they require coordinated, multispecialty treatment. The disaster-exposed group was compared to cases matched 1:4 on diagnosis, year of diagnosis, and socioeconomic status (measured as county-level median income) in 164 counties across 9 SEER registries outside of Louisiana which had not experienced a hurricane or tropical storm during the study period. Using Federal Emergency Management Administration disaster declaration data, ${ }^{2}$ patients were excluded from the comparison group if they resided in areas affected by any other hurricane or tropical storm during the study period. Covariates in our models were cancer stage, age of diagnosis, sex, race/ethnicity, and marital status. The Kaplan-Meier method was used to estimate survival by exposure status, and the exposed versus non-exposed groups were compared using the log-rank test. Cox

Published online October 28, 2019 proportional hazards models were used to examine the association between exposure status and adjust for sociodemographic characteristics and cancer stage. This study was declared exempt from ethical approval as data are publicly available (see data availability statement).

\section{RESULTS}

We identified 1,738 cancer cases diagnosed (794 breast, 532 colorectal, and 412 lung) in parishes affected by Hurricane Katrina and a matched sample of 6557 cancer cases. In unadjusted analyses, exposure to Hurricane Katrina was associated with a $15 \%$ increase in mortality among adults with breast and lung cancer $(\mathrm{HR}=1.15, p$ value $<0.05$ for both) (Fig. 1$)$. With further adjustment for sociodemographic characteristics and cancer stage, associations among all cancer types trended towards higher mortality among cases diagnosed in exposed parishes; however, the only association statistically significant was among breast cancer cases (HR $=1.14,95 \%$ CI 1.00 , 1.32) (Fig. 2).

\section{DISCUSSION}

Our finding that individuals diagnosed with cancer who were exposed to Hurricane Katrina have worse longterm survival is supported by a recent systematic review showing that disasters interrupt the continuity of oncology care. ${ }^{1}$ It is likely that many individuals in our study experienced a disruption in access to healthcare that ultimately affected outcomes. Indeed, treatment disruptions as short as one week have been associated with cancer-related mortality. 4,5

Persistent inequalities affecting cancer progression are well-known to exist in the New Orleans area. Although we matched on key characteristics and account for others in our statistical models, we cannot fully rule out potential residual confounding affecting our results, and the association found between exposure and survival may not be causal, as there could be other confounding variables. However, these adjustments showed consistent findings, albeit with somewhat greater imprecision in the measurement of the confidence interval. Our study examined one 

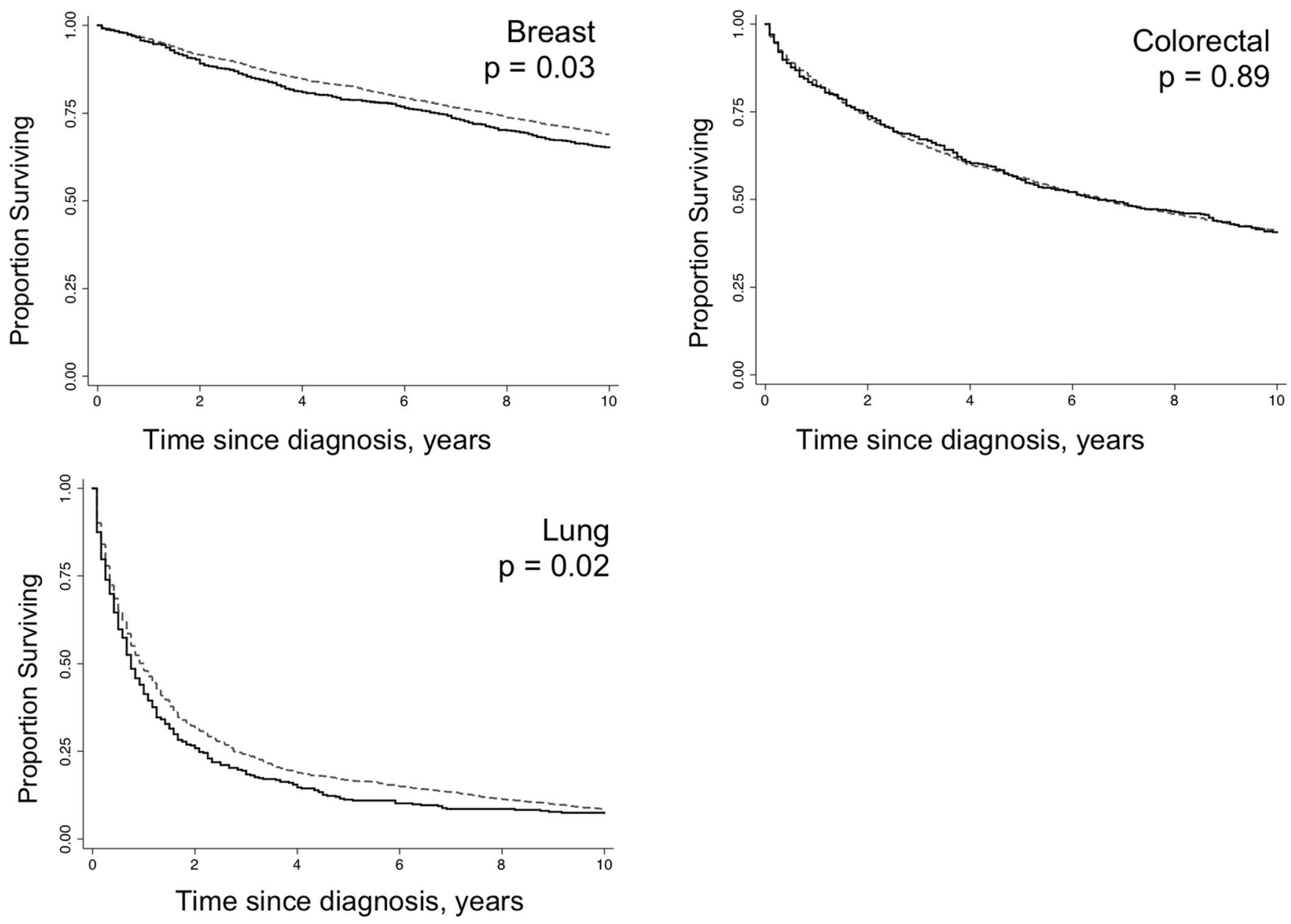

Figure 1 Long-term cancer survival among individuals exposed to Hurricane Katrina. Solid lines represent individuals exposed to hurricane Katrina; dashed lines represent individuals not exposed to Hurricane Katrina. Matched on diagnosis, year of diagnosis, and socioeconomic status (measured as county-level median income). $p$ values obtained from log rank test used to compare survival by exposure status.

single large-scale disaster and found some evidence of this disaster affecting long-term survival. Effects may be different for other health conditions and populations.

Nevertheless, our findings suggest that adverse effects for individuals with cancer may persist well after a disaster is over. There is an urgent need for research investigating the long-term effects of disasters on population health. Indeed, a recent report by the National Academy of Medicine called for the identification of gaps in healthcare system disaster response in order to better promote models of recovery. ${ }^{6}$ To this end, efforts should be extended beyond the acute response period to consider the health needs of those with serious chronic conditions.

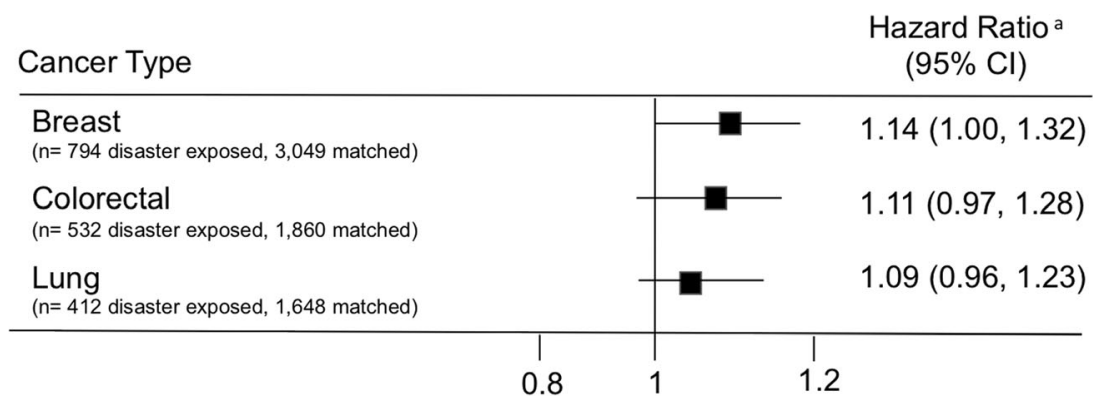

Figure 2 Hazard ratios for the association between exposure status and mortality by cancer type. The superscript lowercase letter "a" means adjusted for age at diagnosis, sex, race, marital status, and cancer stage after matching on diagnosis, year of diagnosis, and socioeconomic status. 
Acknowledgments: Research reported in this publication was supported by the National Institute on Aging of the National Institutes of Health under award number K23AG059890.

Corresponding Author: Sue Anne Bell, PhD, FNP-BC; Institute for Healthcare Policy and Innovation, University of Michigan , Ann Arbor, MI , USA (e-mail: sabell@umich.edu).

Data Availability The data that support the findings of this study are publicly available from https://seer.cancer.gov and https://www. fema.gov/data-feeds.

\section{Compliance with Ethical Standards:}

Conflict of Interest: The authors declare that they do not have a conflict of interest.

Disclaimer: The content is solely the responsibility of the authors and does not necessarily represent the official views of the National Institutes of Health.

\section{REFERENCES}

1. Man, R.X., Lack, D.A., Wyatt, C.E,. \& Murray V. The effect of natural disasters on cancer care: a systematic review. Lancet Oncol 2018;19(9):e482-e499.
2. Federal Emergency Management Agency. Disaster Declarations. 2019; https://www.fema.gov/disasters. Accessed 29 April 2019.

3. Kishore, N., Marqués, D., Mahmud, A., Kiang, M., Rodriguez, I., Fuller, A. et al. Mortality in Puerto Rico after Hurricane Maria. N Engl J Med 2018;379(2): 162-170

4. Hershman, D.L., Shao, T., Kushi, L.H., Buono, D., Tsai, W. Fehrenbacher, L., et al. Early discontinuation and non-adherence to adjuvant hormonal therapy are associated with increased mortality in women with breast cancer. Breast Cancer Res Treat 2011;126(2):529-537.

5. Ganesan, P., Sagar, T.G., Dubashi, B., Rajendranath, R., Kannan, K. Cyriac, $\mathbf{S}$,. et al. Nonadherence to imatinib adversely affects event free survival in chronic phase chronic myeloid leukemia. Am J Hematol 2011:86(6):471-474.

6. Committee on Post-Disaster Recovery of a Community's Public Health M, and Social Services Board on Health Sciences Policy Institute of Medicine. Healthy, Resilient, and Sustainable Communities After Disasters: Strategies, Opportunities, and Planning for Recovery. Washington, DC: National Academy of Sciences; 2015.

Publisher's Note Springer Nature remains neutral with regard to jurisdictional claims in published maps and institutional affiliations. 